\title{
Examination of Reduced Order Building Models with Different Zoning Strategies to Simulate Larger Non-Residential Buildings Based on BIM as Single Source of Truth
}

\author{
David Jansen $^{1} \quad$ Veronika Richter $^{2} \quad$ Diego Cordoba Lopez ${ }^{1}$ Philipp Mehrfeld $^{1}$ Jérôme Frisch ${ }^{2}$ \\ Dirk Müller ${ }^{1}$ Christoph van Treeck ${ }^{2}$ \\ ${ }^{1}$ Institute for Energy Efficient Buildings and Indoor Climate, E.ON Energy Research Center, RWTH Aachen \\ University, Germany, \{david.jansen, diego.cordoba, pmehrfeld, \\ dmueller\}eoonerc.rwth-aachen. de \\ ${ }^{2}$ Institute of Energy Efficiency and Sustainable Building E3D, RWTH Aachen University, Germany, \\ \{richter, frisch, treeck\}@e3d.rwth-aachen.de
}

\begin{abstract}
Non-residential buildings are accountable for $11 \%$ of global energy-related $\mathrm{CO} 2$ emissions (United Nations Environment Programme 2018). To increase the performance in this sector, Building Energy Performance Simulation (BEPS) is one feasible approach. Therefore, there is need for reliable and fast simulation models. One feasible approach are so called Reduced Order Models (ROMs). Thus in this paper, a comparison between the results of the established BEPS tool EnergyPlus and a ROM in Modelica with a reduced number of resistances and capacities is applied at the use case of a non-residential building. A self-developed toolchain was used to create equal models for ROM and EnergyPlus based on the same Building Information Modeling (BIM) model. The comparison shows that the reduced model deviates by $\pm 10 \%$ in annual heating and cooling. To increase accuracy and decrease computational effort the zoning strategy of non-residential buildings is investigated. The investigation shows that using a suitable zoning approach can reduce the computational effort by up to $97 \%$.
\end{abstract}

Keywords: BEPS, BIM, zoning, reduced order, ROM

\section{Introduction}

The simulation of larger non-residential buildings is an important aspect in the field of building simulations but comes with additional challenges, compared to the simulation of smaller buildings like single-family houses. Three major challenges are:

(i) Higher effort for creation and parametrization of the simulation model

(ii) Higher computational effort to solve the resulting system of equations

(iii) Necessity of zoning the simulation model due to higher influences of different room usages compared to residential buildings
Challenges (i) and (ii) can be addressed by using simplified ROMs combined with statistical data enrichment. One tool that offers these features is TEASER (Remmen et al. 2018) which provides the capability to create Modelica models based on Python code and the open-source Modelica library AixLib (Müller et al. 2016). By using Modelica, the resulting simulation models provide a huge amount of flexibility to integrate new functions and coupling the resulting BEPS models with Heating, Ventilation and Air Conditioning (HVAC) models. Challenge (iii) can be addressed by abiding existing rules of thumb but this often leads to more effort due to time consuming manual operations. The general problem that comes with zoning in BEPS is that falsely zoned models can have localized unrealistic peaks in heating and/or cooling demand. This happens due to over-discretization of zones, where e.g. the irradiated heat of high solar gains is not correctly passed to nearby zones. (Dogan, Reinhart, and Michalatos 2016; Smith, Bernhardt, and Jezyk 2011)

This paper focuses on two questions: first, in which cases using the ROM approach is suitable for simulating larger non-residential buildings, and second, how different zoning strategies affect the accuracy and computational effort. To investigate the first question, two simulation models of the same building are created by using BIM as a Single Source of Truth (SSOC) to guarantee the similarity of the models. One model is a ROM model based on the German guideline VDI 6007 -1 (2015) created with TEASER, the other is an EnergyPlus simulation model. The results of these models are compared and put into the context of previous research results. The second question is answered by applying different levels of zone reduction on the TEASER model based on existing research and investigating the results. Thereby, the special case of the used multi-zone model which uses adiabatic inner walls is also taken into account. 


\section{Related Work}

\subsection{Model Comparison}

Initial verification of the ROM was already done by Lauster, Constantin, and Remmen (2017) based on the ASHRAE 140 (ASHRAE 2017) test cases. The same test cases were also simulated with EnergyPlus (Henninger, Robert H. and Witte, Michael J. 2015). These two studies showed that both the EnergyPlus model and the ROM are delivering results that are inside the boundaries for most of the test cases. In comparison to the other simulation tools, EnergyPlus tends to predict comparatively low annual heating demands. The ROM tends to predict too high fluctuations in the indoor temperatures and an underestimation of thermal mass for low mass buildings and vice versa an overestimation and too damped behavior for heavy mass buildings (Lauster, Constantin, and Remmen 2017). Kuniyoshi, Kramer, and Lindauer (2018) performed a comparison of EnergyPlus and a 6 resistance and 4 capacities model which uses different capacities for zone air, inner walls, outer components, and floors for a singlefamily house. They found that the default VDI 6007-1 model performs well for representing the conductive and convective heat transfer but has problems with the correct representation of solar irradiation. This challenge was met by the usage of a curve fitting approach instead of an areaweighted approach for the determination of the distribution factors for internal solar gains. All the listed works deal with test cases for buildings in the size of one room up to single-family houses. Therefore in this paper, an investigation of a four-storey non-resident building with mainly office usage is performed.

\subsection{Zoning in BEPS}

The German standard DIN V 18599-1 (2018) provides guideline values for the zoning of buildings divided into three aspects. (i) The usage type of the respective zone, (ii) the type of conditioning, and (iii) the glazing ratio, where a distinction is made between $25 \%$ and $75 \%$ glazing ratio. The impact of different zoning strategies was investigated by Brès et al. (2017) by applying different zoning strategies on multiple floor plans but not whole buildings. They covered perimeter and core distinctions, usage type distinctions, orientations of the zones, and finally combined these strategies. They conclude that the zoning affects the simulation results in a wide range based on used strategy and building. Especially simple approaches like perimeter core distinctions resulted in deviations up to $30 \%$, whereas the combination of different zoning strategies led to $<10 \%$ of deviations in total heating load. Additional to research regarding how to zone buildings and how this affects the simulation results, multiple studies were performed regarding automatic zoning. E.g. Dogan, Reinhart, and Michalatos (2016) as well as Smith, Bernhardt, and Jezyk (2011) presented automatic approaches for zoning for complex building shapes based on algorithms with in-depth analysis. How-

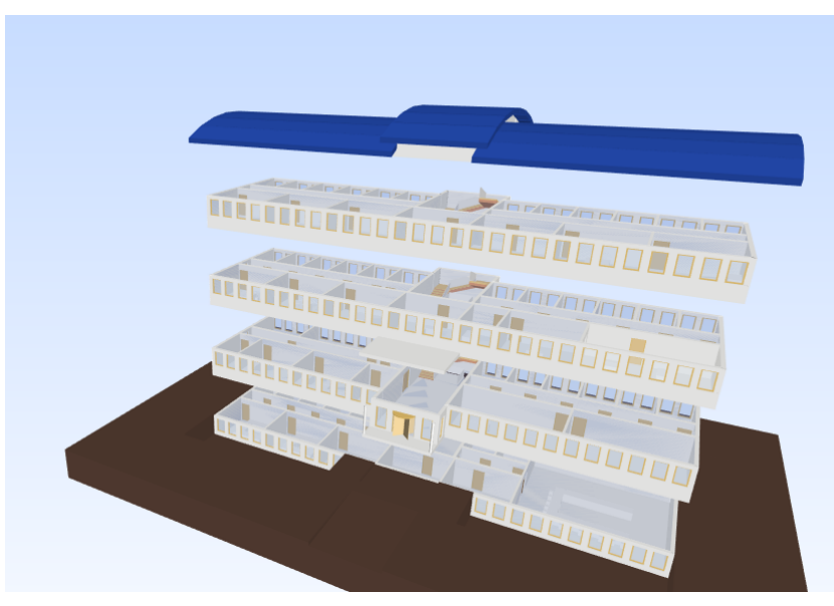

Figure 1. IFC of KIT Office Building fig. 1

ever, both approaches are valid only for the early concept phase of the building, where interior space divisions are still undefined. Another approach was developed by Georgescu, Eisenhower, and Mezic (2012). They used the Koopman operator to analyze the temperature behavior in different rooms during building simulation to carry out optimal zoning strategies. The approach shows promising results but a prior simulation of the detailed and not zoned model is mandatory. The performed test cases in the work carried out some guidelines which confirm the already mentioned rules and add the additional rule that small volume and surface areas can be merged to much larger adjacent zones with little loss of accuracy.

Both, the comparison of ROMs and established simulation tools, as well as general investigation of zoning on simulations models, were already discussed in existing research. The special cases addressed by this paper are large non-residential buildings and the zoning of reduced ROMs using BIM models as a source.

\section{Methodology}

\subsection{Defining the Model Setup}

TEASER and EnergyPlus both in their core are based on a resistance-capacity-based approach to represent the underlying physical concepts (U.S. Department of Energy 2020; Remmen et al. 2018). However, EnergyPlus uses a much more detailed approach, while TEASER uses a reduced order approach. As EnergyPlus is an established simulation tool, the underlying assumptions will not be discussed in detail and the reader is referred to U.S. Department of Energy (2020) for further details. While TEASER is capable of exporting different types of models in this paper the two capacity model based on VDI $6007-1$ (2015) is used. Compared to the original guideline model it extended by an additional resistance for windows. Lauster, Müller, and Nytsch-Geusen (2018) already showed that this configuration is predicting the thermal behavior well for residential buildings. The periodic penetration depth that defines which part of the wall will be 
used as capacity and resistance and which part will be used only as resistance is set to five days according to Lauster, Müller, and Nytsch-Geusen (2018). All simulations were performed in Dymola using the DASSL solver with a tolerance of $10 E^{-4}$. Both models use the test reference year of 2012 for Aachen, Germany (Lawrie and Crawley 2019).

\subsection{Use Case}

The investigations in this paper are applied to the Industry Foundation Classes (IFC) file KIT Office Building (Haefele, Karl-Heinz 2021) shown in Figure 1. This model is chosen because it represents a non-residential building with more complex geometry and different types of rooms, such as conference rooms, single and group offices, and laboratories. The model was created by Archi$C A D 20$ and offers good quality regarding semantic data and especially regarding $2^{\text {nd }}$ level Space Boundaries (SB). Even if the used IFC file provides a comparatively good quality regarding semantics and $\mathrm{SB}$, the thermal properties, layer structure of the building elements and the occupancy-related information is not completely present. To overcome this and to guarantee that both models, EnergyPlus and the ROM, rely on the same data, the building information is enriched by existing data. This data is based on the templates stored in the current release of TEASER ${ }^{1}$.

For building physics related data, the physics for a typical building with a construction year between 1995 and 2015 and a light building structure is assumed. For occupancyrelated information, the room names in the IFC are used to identify the occupancy type. Based on these types and the mentioned templates, the required information for the simulation is set. Additionally, the conditions displayed in Table 1 are applied. A constant infiltration rate $\left(n_{\text {inf }}\right)$ and the same solar absorption coefficient $\left(\alpha_{\text {solar,abs }}\right)$ for the materials are used.

For the comparison between EnergyPlus and the ROM, no internal gains are applied to reduce the influences of simulation software-related interpretation of the internal loads and thereby focus on the comparison of the simulated building physics.

Table 1. Additional conditions.

\begin{tabular}{llll}
\hline$T_{\text {set,cooling }}\left[{ }^{\circ} \mathrm{C}\right]$ & $T_{\text {set,heating }}\left[{ }^{\circ} \mathrm{C}\right]$ & $n_{\text {inf }}[1 / \mathrm{h}]$ & $\alpha_{\text {solar,abs }}$ \\
\hline 25 & 20 & 0.2 & 0.7 \\
\hline
\end{tabular}

\subsection{Comparing TEASER and EnergyPlus}

Using BIM as SSOC allows using different tools and approaches to create simulation models that reflect the same building.

In a suitable modeled BIM model for BEPS, all building physics and almost all simulation relevant information including profiles for internal gains can be included

\footnotetext{
${ }^{1}$ https://github.com/RWTH-EBC/TEASER/tree/development commit $95243 d 4$
}

Table 2. Four criteria for zoning.

\begin{tabular}{l|l}
\hline Perimeter/Core (PC) & $\begin{array}{l}\text { Internal zone } \\
\text { External zone }\end{array}$ \\
\hline Orientation (O) & $\begin{array}{l}\text { North/East } \\
\text { South/West }\end{array}$ \\
\hline & $<30$ \\
& $30<G R<50$ \\
Glazing Ratio (GR) [\%] & $50<G R<70$ \\
& $>70$ \\
\hline Usage (U) & $*$ \\
\hline
\end{tabular}

Table 3. Zoning Setups.

\begin{tabular}{|c|c|c|}
\hline Approach & $n_{\text {Zones }}$ & Zoning \\
\hline $\mathrm{PC}$ & 2 & \\
\hline $\mathrm{PC}+\mathrm{O}$ & 3 & \\
\hline $\mathrm{U}$ & 6 & \\
\hline $\mathrm{PC}+\mathrm{O}+\mathrm{U}$ & 9 & \\
\hline $\mathrm{PC}+\mathrm{O}+\mathrm{U}+\mathrm{GR}$ & 12 & \\
\hline
\end{tabular}

by using the non-proprietary IFC format (buildingSmart 2021). As the conversion of the BIM model to simulation model in terms of BEPS is still a subject of research, the self-developed toolchain BIM2SIM (Jansen, David et al. 2021) for creating simulation models of different domains is used in this paper. Besides the creation of Computational Fluid Dynamics (CFD) and HVAC simulation models, it allows also BEPS simulations with the tool TEASER (Remmen et al. 2018) and EnergyPlus (EnergyPlus 2020). As even BIM-Models created in the re- 


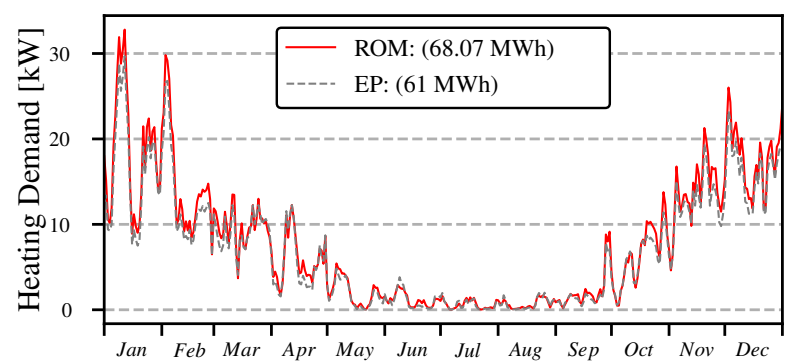

(a) Annual heating demand

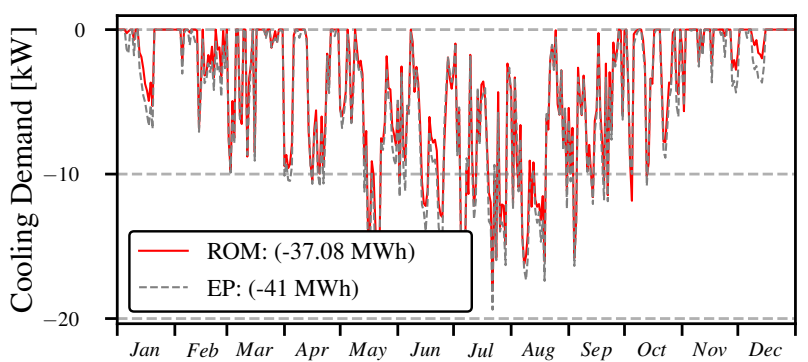

(b) Annual cooling demand

Figure 2. Annual heating (a) and cooling (b) demands.

search context are still lacking some of the information needed for BEPS, enrichment methods of BIM2SIM are used to create enriched simulation models. Using the combination of the BIM model as a SSOC and the modular toolchain, BIM2SIM allows creating twin models with the same parametrization and same boundary conditions in TEASER and EnergyPlus. As TEASER exports a Modelica multi-zone model in which all thermal zones have adiabatic inner walls, no inter-zonal heat transfer is taken into account. To provide a valid comparison, the EnergyPlus model is configured with adiabatic inner walls and without infiltration between the zones as well. In both models, every room is represented by a single zone which leads to 82 zones in total.

\subsection{Zoning}

After the comparison of TEASER with EnergyPlus, in the next step different zoning strategies are applied to investigate the influence on computational effort and accuracy. The reference is the not zoned model where each of the 82 rooms is represented by one separate zone. Based on the related work, the reference case will be zoned by using different combinations of the four criteria shown in Table 2 . The glazing ratio is divided into 4 groups, deviating from the recommendation of DIN 18599-1.

This results in 5 separate options to zone the building, which are displayed in Table 3 in order of increasing number of criteria and thus zones. The most detailed one is a $n_{\text {Zones }}=12$ setup where all criteria are taken into account. To merge zones of different usage, the corresponding geometries and conditioning attributes have to be averaged. In general, this information can be divided into extensive attributes likes wall areas and air volumes, intensive attributes like temperature set points, boolean attributes, and lists, like occupancy profiles. The needed functions are implemented into the algorithms to automate the process and minimize errors. The resulting methodologies are transferred into algorithms and included in the BIM2SIM toolchain. This offers the advantage that the zoning has no more to be done manually, but can be completely automated based on the information in the corresponding BIM model and thereby integrated into the workflow.

\section{Results and Discussion}

\subsection{Comparing TEASER and EnergyPlus}

The resulting models and their simulation results are compared loosely based on the methodology of the ASHRAE 140 specifications (ASHRAE 2017).

The yearly time series data for heating and cooling, including the annual heating and cooling demands, are compared in Figure 2. Comparing the results shows that both, heating and cooling dynamics on the building level are quite similar. However, the ROM tends to slightly higher peak demands for heating and lower peak demands for cooling. Also, the annual consumption for heating of the $\mathrm{ROM}$ is $10 \%$ higher and the annual consumption for cooling $10 \%$ lower compared to EnergyPlus. Comparing these results with the existing ASHRAE 140 verifications for Energyplus indicates that EnergyPlus is on the lower end of the allowed bandwidth regarding heating (Henninger, Robert H. and Witte, Michael J. 2015). The behavior that the ROM predicts higher heating and lower cooling loads can also be found in the verification made by Lauster, Constantin, and Remmen (2017).
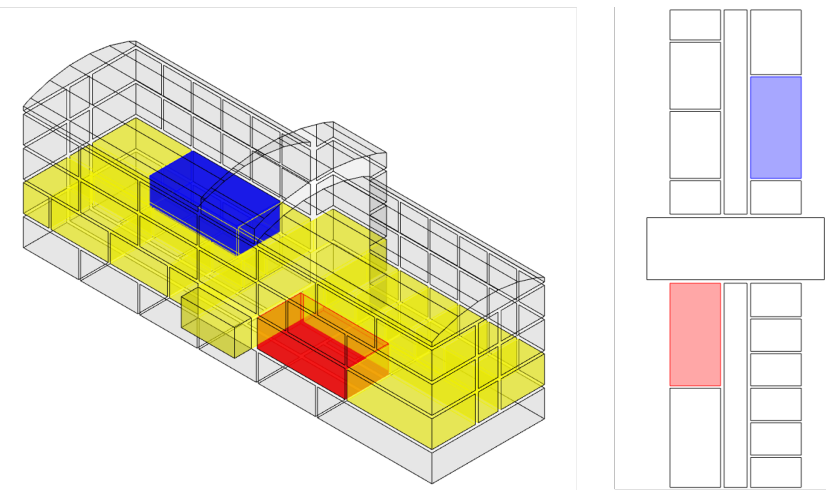

Figure 3. Selected rooms for in depth analysis.

For the following investigations, two rooms are selected to be examined more closely. The rooms are highlighted in Figure 3, whereas the red one is orientated towards the south and the blue one towards the north. Both rooms have a glazing ratio $60 \%<G R<70 \%$. In Figure 4 the inner temperatures of the two rooms are shown on a daily basis for a winter and a summer day, while the building is 


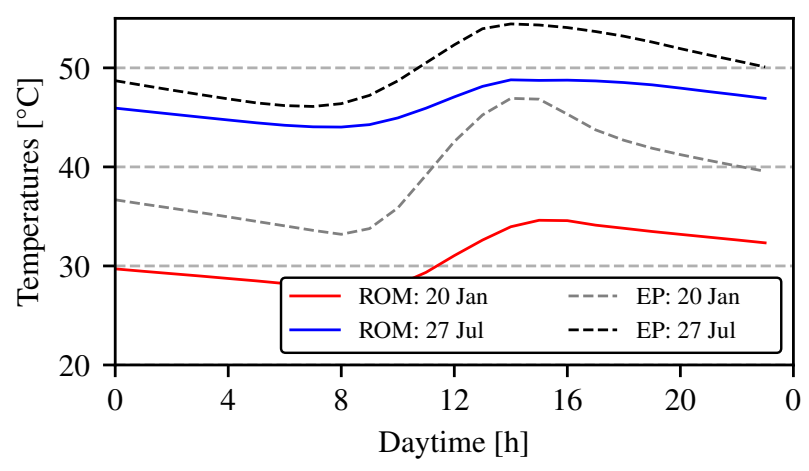

(a) south-orientated room

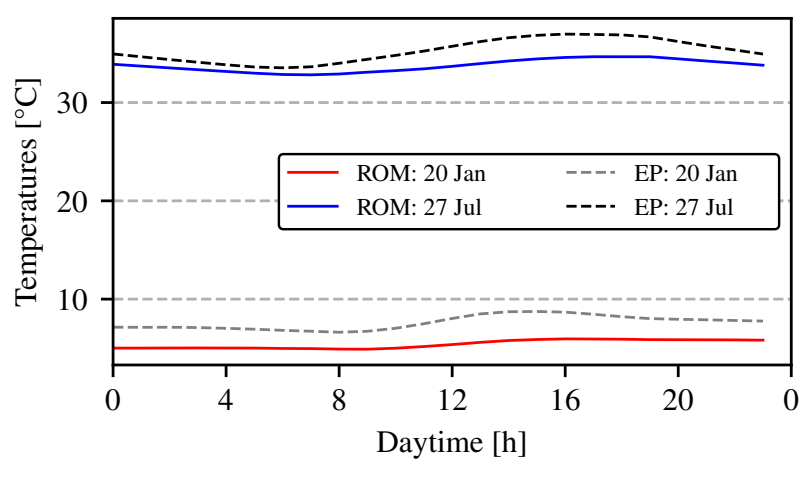

(b) north-orientated room

Figure 4. Free floating temperature for two days for a south- (a) and north- (b) orientated room.

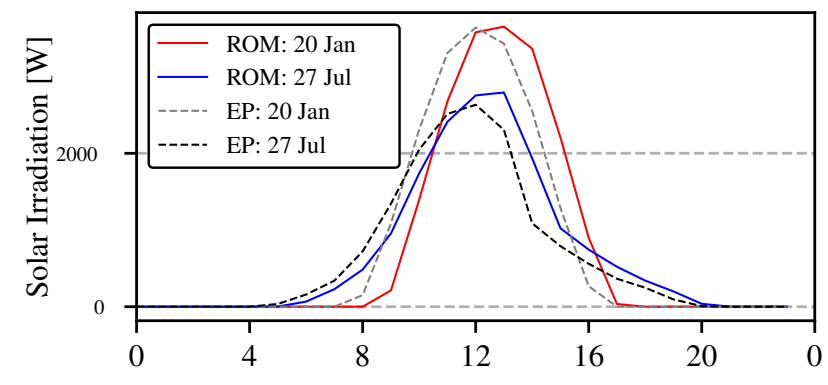

(a) south-orientated room

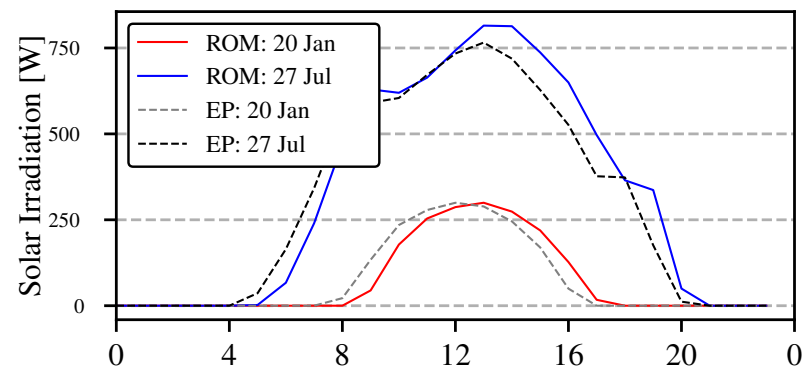

(b) north-orientated room

Figure 5. Solar irradiation through windows for both rooms and different days.

free-floating without heating or cooling provided. In both cases, the ROM results in lower temperatures. This behavior is more intense for the south-orientated room. The qualitative timeseries is anyhow described in the same way by EnergyPlus and ROM. The high temperatures even in winter for the south-orientated room show the effect of over-discretization for the special case of adiabatic inner walls. Due to the high glazing ratio, the temperatures in the room rise up to $48^{\circ} \mathrm{C}$ in the reference case of EnergyPlus while the north-orientated room stays below $10^{\circ} \mathrm{C}$. In Figure 5 the solar irradiation through windows for both rooms is compared for the two different days. The ROM reacts slower to the changes in irradiation as the EnergyPlus simulation does. Apart from that the solar irradation is calculated similar for both models.

In Figure 6 the heating and cooling powers for the two rooms and the same winter and summer days are shown. The time series of the ROM and EnergyPlus have the same qualitative behavior, whereby the ROM shows again the higher peak demands and reacts a bit slower. The effect of over-discretization is again visible as the south-orientated room needs to be cooled even in winter.

It was shown that the ROM predicts the annual heating and cooling in the range of $\pm 10 \%$ and also the dynamic results for the whole building are deviating only slightly from the EnergyPlus predictions. Comparing these values with the literature indicates that there are large variations in simulation results even while simulating the same building. (Choi 2017) However, on a daily basis and when investigating the detailed behavior of single rooms, the ROM shows bigger deviations. Due to the assumption that both models have adiabatic inner walls, unrealistic temperatures occur in south-orientated rooms with a high glazing ratio. It can be concluded, that, especially for investigations on a daily basis or room level, the assumption of adiabatic inner walls has to be evaluated carefully.

\subsection{Comparing zoning strategies}

The results must be evaluated concerning two questions:

(i) How to improve the computational effort without losing accuracy compared to the reference case?

(ii) How can zoning be used to reduce the influences of assuming adiabatic interior walls?

The comparison of the reference setup with $n_{\text {Zones }}=82$ and the 5 different zoning setups introduced in Table 3 are displayed in Figure 7 . The total consumption for heating and cooling is shown in total as well as the relative deviation from the reference case. Additionally, the used CPU-time for calculation is shown on a logarithmic basis. It is recognizable that especially the zoning strategies with a low number of zones have a high impact on the resulting consumption. However, the two most detailed strategies with $n_{\text {Zones }}=9$ and $n_{\text {Zones }}=12$ have only a deviation of around $1 \%$ from the reference case while having a remarkable advantage in computation time. This suggests that for the use case of the here considered office building 


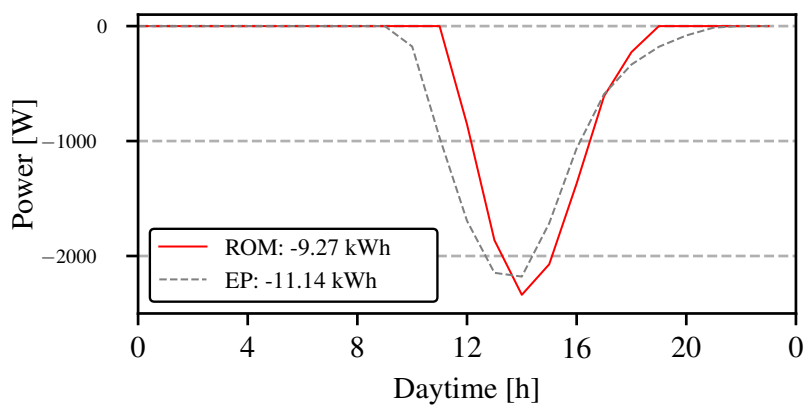

(a) south-orientated room (20. Jan.)

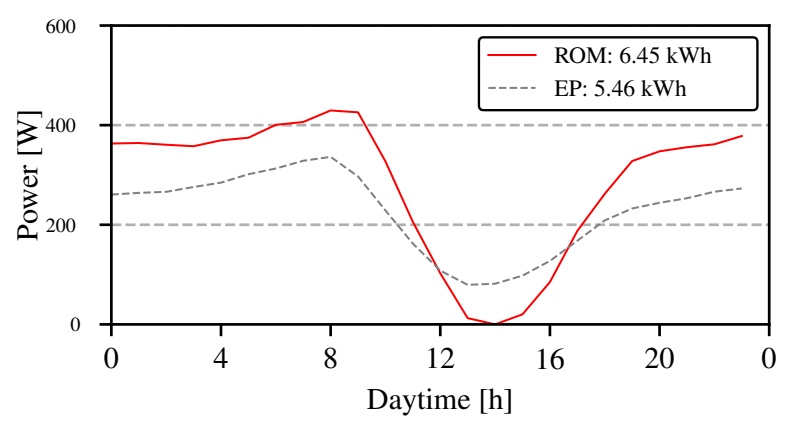

(c) north-orientated room (20. Jan.)

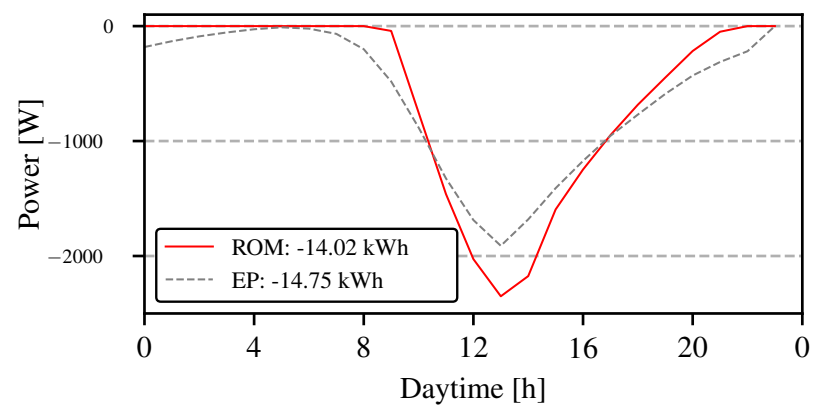

(b) south-orientated room (27. Jul.)

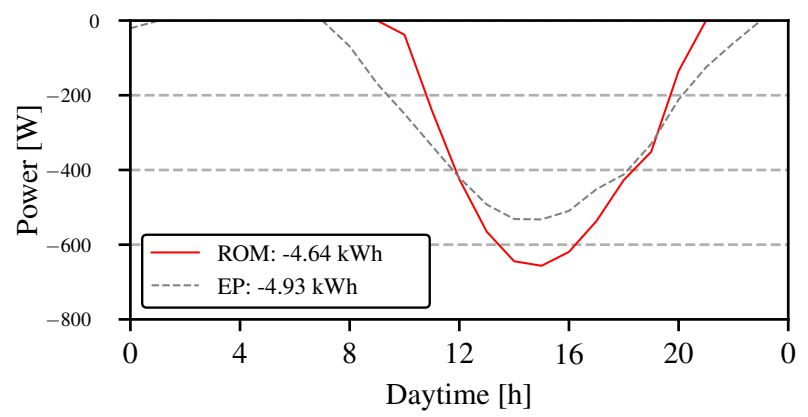

(d) north-orientated room (27. Jul.)

Figure 6. Time series for heating and cooling for both rooms and different days.

the consideration of the glazing ratio does not make a decisive difference. The computational effort can be reduced by up to $97 \%$. It can be concluded that regarding question (i) using the $n_{\text {Zones }}=9$ or $n_{\text {Zones }}=12$ approach leads to a drastic reduction in computational effort while the results remain quite similar. To prove that also the dynamics are not changing, in Figure 8 the comparison between the $n_{\text {Zones }}=9$ and $n_{\text {Zones }}=12$ approach is shown on an annual basis.

To investigate question (ii) as a first step, EnergyPlus was used again to simulate the building but without the assumption of adiabatic inner walls. The results show that the simulated heating consumption decreases by $7.5 \%$ and the cooling consumption by $22 \%$ compared to the values shown in Figure 2 when the inter zonal heat transfer is taken into account. Comparing these results with the results in Figure 7 makes it clear that a smaller number of zones with a combination of south-orientated and northorientated rooms like for the $n_{\text {Zones }}=6$ variant, which uses only the usage for aggregation, leads to a more realistic re-
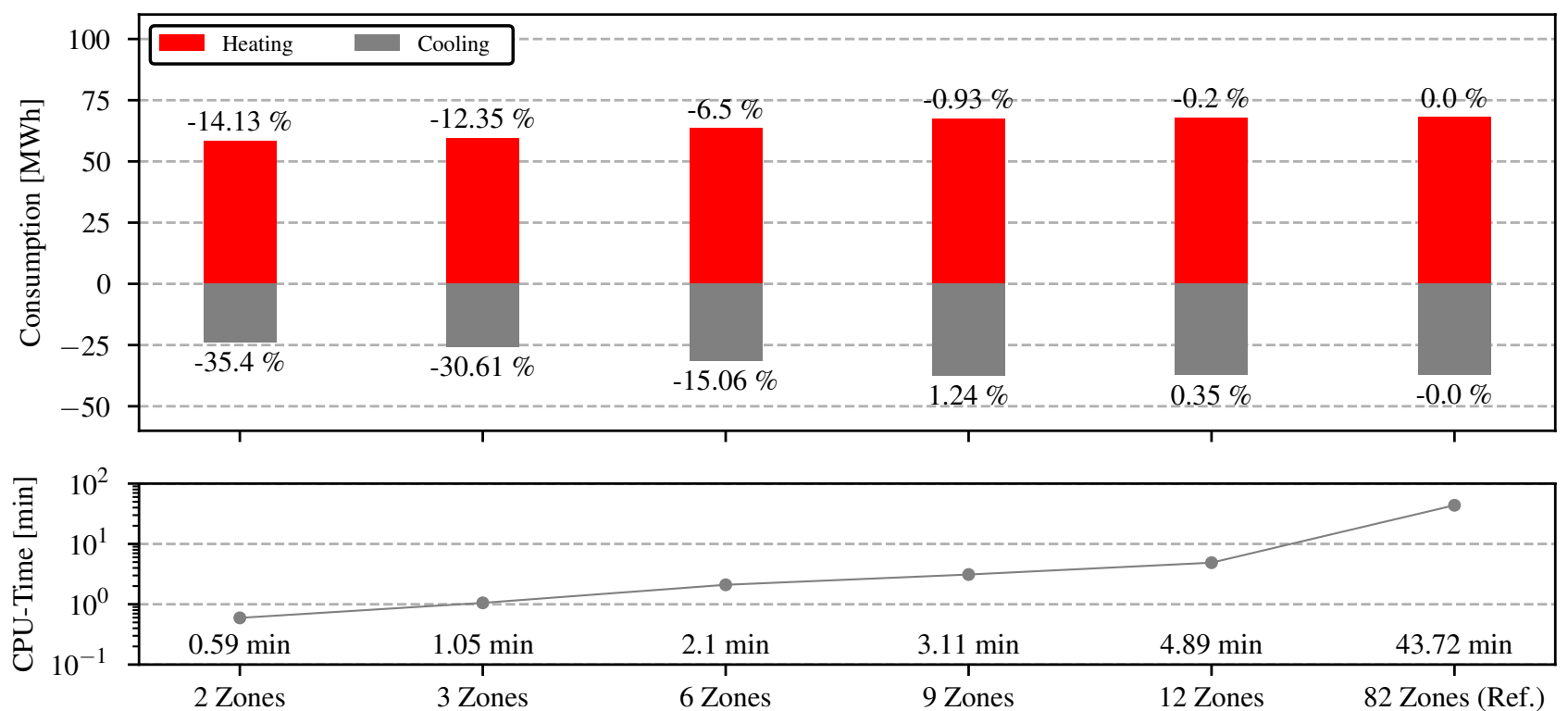

Figure 7. Consumption for the 5 zoning strategies and the reference case including relative deviation to reference case. 


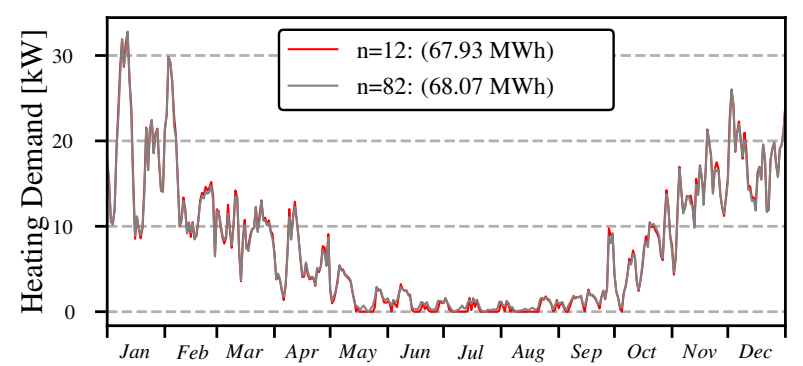

(a)

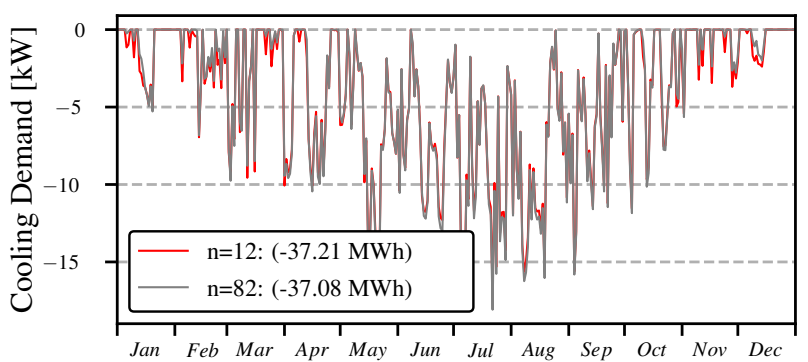

(b)

Figure 8. Annual heating (a) and cooling (b) demands for not zoned and zoned building with 12 zones.

sult. Therefore, it can be concluded that for ROMs, where the assumption of adiabatic inner walls is made, the most detailed approaches, where the orientation is taken into account, leads again to an over-discretization. In these cases, zoning strategies with a lower number of zones should be preferred.

\section{Conclusion}

The provided research performs a first investigation on comparing the simulation of complex buildings with a ROM approach against an established simulation program. By using EnergyPlus it was shown that even reduced order approaches deliver good qualitative and quantitative results and are capable to represent the overall thermal behavior of the building. Nevertheless, it was also shown that not all dynamics can be covered by the ROM. The reduced model tends to have lower temperatures and therefore higher heating and lower cooling loads, both in annual as peak demand. It can be concluded that for the investigated case of a light constructed non-residential building with a total glazing ratio of $25 \%$ the ROM is predicting the annual heating and cooling loads, as well as the peak demands similar to the reference model in EnergyPlus within a range of $10 \%$. If a detailed analysis of the thermal behavior on shorter periods is wanted, one should consider using a detailed approach instead of a reduced approach. For the analysis on a yearly basis the reduced model predicts the behavior in reasonable ranges.

Furthermore, it was shown that by reducing the number of zones the computational effort can be drastically reduced compared to the not zoned building. Moreover, the reduction of the building on a really small number of zones can lead to slight to medium differences in the calculated heating loads and massive differences regarding cooling loads. For the special assumption of adiabatic inner walls used by the investigated ROM, a suiting zoning strategy can reduce the unintentional effect of too high heating and cooling demands. The investigated zoning strategies were implemented into the BIM2SIM toolchain so that they can be easily applied to new buildings in future. In future work, the comparison between TEASER and EnergyPlus should be done with internal gains and dynamic infiltration rates to cover more fluctuating changes. These changes can have different excitation frequencies which could pose an additional challenge for the reduced approach as the number of excitation frequencies that can be covered is determined by the number of capacities and resistances (lauster_verication_2017). Furthermore, the comparison should be extended to multiple buildings with different geometry and mass classes. Thereby, the insights of this paper can be verified. Additionally, a deeper investigation of the reasons for the over proportional increasing computational effort by the number of zones should be performed.

\section{Acknowledgements}

The authors gratefully acknowledge the financial support of the German Federal Ministry for Economic Affairs and Energy in the project "BIM2SIM" (project number 03ET1562A). The authors also want to thank for the support and discussions inside the IBPSA Project 1.

\section{References}

ASHRAE (2017). ANSI/ASHRAE Standard 140:2017, Standard method of test for the evaluation of building energy analysis computer programs. Tech. rep. Atlanta, USA: American Society of Heating, Refrigerating and Air-Conditioning Engineers.

Brès, Aurélien et al. (2017). "Impact of zoning strategies for building performance simulation". In: Proceedings of a meeting held 10-12 July 2017, Nottingham, UK. Nottingham, UK.

buildingSmart (2021). IFC4 Documentation. https://standards. buildingsmart.org/ifc/dev/ifc4_2/final/html/. (Visited on 2021-05-10).

Choi, Joon-Ho (2017). "Investigation of the correlation of building energy use intensity estimated by six building performance simulation tools". In: Energy and Buildings 147, pp. 14-26. ISSN: 03787788. DOI: 10.1016/j. enbuild. 2017. 04.078 .

DIN V 18599-1 (2018). Energetische Bewertung von Gebäuden - Teil 1: Allgemeine Bilanzierungsverfahren, Begriffe, Zonierung und Bewertung der Energieträger.

Dogan, Timur, Christoph Reinhart, and Panagiotis Michalatos (2016). "Autozoner: an algorithm for automatic thermal zoning of buildings with unknown interior space definitions". en. In: Journal of Building Performance Simulation 9.2. Number: 2, pp. 176-189. ISSN: 1940-1493, 1940-1507. DOI: 10.1080/ 19401493.2015.1006527. 
EnergyPlus (2020). www.energyplus.net.

Georgescu, Michael, Bryan Eisenhower, and Igor Mezic (2012). "Creating zoning approximations to building energy models using the Koopman operator". In: IBPSA USA Sim Build.

Haefele, Karl-Heinz (2021). KIT IFC Examples - IfcWiki. https: $/ / w w w$.ifcwiki.org/index.php? title = kit_ifc_examples. (Visited on 2021-05-07).

Henninger, Robert H. and Witte, Michael J. (2015). EnergyPlus 8.3.0-b45b06b780 Testing with Building Thermal Envelope and Fabric Load Tests from ANSI/ASHRAE Standard 1402011. Tech. rep.

Jansen, David et al. (2021). "BIM2SIM - Development of semiautomated methods for the generation". In: Building Simulations 2021 - to be published, accepted for presentation. Bruges, Belgien.

Kuniyoshi, Ryuta, Michael Kramer, and Manuel Lindauer (2018). "Validation of RC Building Models for Applications in Energy and Demand Side Management". en. In: eSIM 2018 Conference Proceedings. Montréal, Canada, p. 10.

Lauster, Moritz, Ana Constantin, and Peter Remmen (2017). "Verification and Comparison of High and Low Order Building Models from the Modelica Library AixLib using ASHRAE Standard 140". In: Proceedings of Building Simulation 2017. San Francisco, USA: E.ON Energy Research Center, RWTH Aachen University.

Lauster, Moritz, Dirk Müller, and Christoph Nytsch-Geusen (2018). "Parametrierbare Gebäudemodelle für dynamische Energiebedarfsrechnungen von Stadtquartieren". PhD thesis. Aachen. ISBN: 978-3-942789-59-2.

Lawrie, Linda K. and Drury B. Crawley (2019). Development of Global Typical Meteorological Years (TMYX). http://climate. onebuilding.org.

Müller, Dirk et al. (2016). "Aixlib - An Open-Source Modelica Library Within the Iea-Ebc Annex 60 Framework". In: Conference Proceedings of Central European Symposium on Building Physics. Dresden, Germany.

Remmen, Peter et al. (2018). "TEASER: an open tool for urban energy modelling of building stocks". In: Journal of Building Performance Simulation 11. DOI: 10.1080/19401493.2017. 1283539.

Smith, Lillian, Kyle Bernhardt, and Matthew Jezyk (2011). "Automated energy model creation for conceptual design". In: Proceedings of the 2011 Symposium on Simulation for Architecture and Urban Design. SimAUD '11. Boston, Massachusetts: Society for Computer Simulation International, pp. 13-20.

U.S. Department of Energy (2020). EnergyPlus: Engineering Reference Version 9.4.0. Tech. rep.

United Nations Environment Programme (2018). Global Status Report 2018 - Towards a zero-emission, efficient and resilient buildings and construction sector. Tech. rep. ISBN: 97892-807-3729-5. Paris, France: International Energy Agency (IEA).

VDI 6007 -1 (2015). Calculation of transient thermal response of rooms and buildings - Modelling of rooms. 